\title{
Author Correction: The interferon-inducible isoform of NCOA7 inhibits endosome- mediated viral entry
}

Tomas Doyle, Olivier Moncorgé, Boris Bonaventure, Darja Pollpeter (D), Marion Lussignol, Marine Tauziet, Luis Apolonia (D), Maria-Teresa Catanese, Caroline Goujon (D) and Michael H. Malim (D)

Correction to: Nature Microbiology https://doi.org/10.1038/s41564-018-0273-9, published online 26 November 2018.

In the version of Supplementary Fig. 5a originally published with this Letter, the authors mistakenly duplicated images of LAMP1 staining in place of CD63 staining; this has now been amended to the correct version shown below.

a)
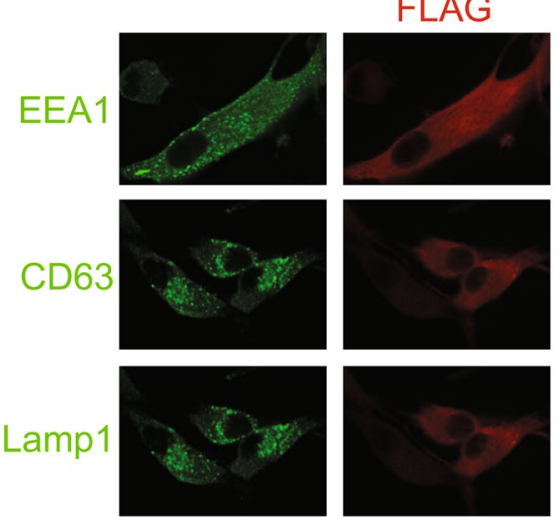

merge
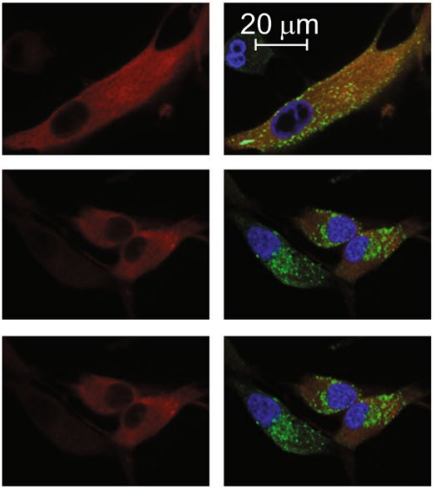

a)
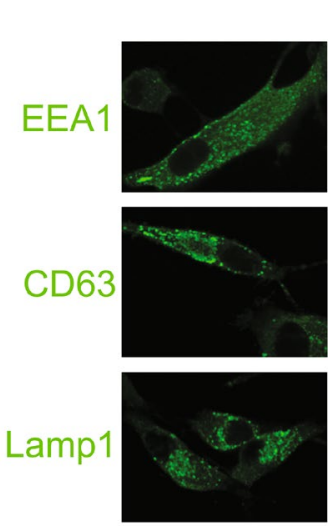

Corrected
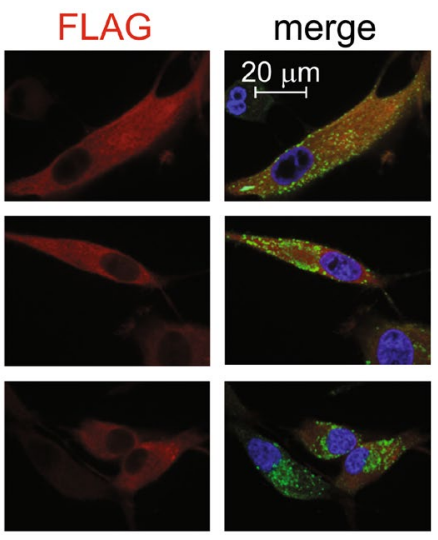

Supplementary Fig. 5a | Orginal and Corrected. 